\title{
Coriocarcinoma gástrico primario: reporte de caso
}

Fernando Barreda Bolaños* 1,a; Humberto Liu Bejarano 1,a; Jéssica Alférez Andía 2,b; Roxana Inoñan García 3,b; Henry Guerra Miller ${ }^{1, c}$; Eduardo Payet Meza ${ }^{1, \mathrm{~d}}$

\section{RESUMEN}

El coriocarcinoma gástrico primario (CGP) es un tumor infrecuente. Debido a su rareza, su patogenia aún no es clara. El diagnóstico se basa en la inmunohistopatología, la cual es positiva para gonadotropina coriónica humana (hCG). Es una neoplasia altamente invasiva y de rápida diseminación hematógena, lo que conlleva a un pobre pronóstico. Se presenta el caso de una paciente mujer de 57 años con hemorragia digestiva alta asociada a baja de peso. La gastroscopia reporta, a nivel de cardias y región paracardial, lesión polipoidea ulcerada de $5 \times 4 \mathrm{~cm}$, con bordes circunscritos y sin presencia de infiltración de pared adyacente, con estigmas de sangrado reciente y anatomía patológica que informa neoplasia maligna pleomórfica ulcerada con inmunohistoquímica positiva para panqueratina. Ante la sospecha de carcinoma poco diferenciado y sin evidencia de metástasis, la paciente fue sometida a gastrectomía total y la patología fue compatible con coriocarcinoma. Por ello, posterior a la cirugía, se realiza estudio de hCG sérico con resultado de $714 \mathrm{mlU} / \mathrm{ml}$, lo que confirmó aún más el diagnóstico. Se decide tratamiento adyuvante con quimioterapia y se realiza seguimiento tomográfico y serológico de hCG sin presencia de enfermedad activa.

Palabras clave: Cáncer gástrico; Coriocarcinoma; Gastrectomía; Gonadotropina coriónica; Cirugía (Fuente: DeCS BIREME).

\section{Primary gastric choriocarcinoma: a case report}

\section{ABSTRACT}

Primary gastric choriocarcinoma (PGC) is an uncommon tumor. Due to its rarity, its pathogenesis is still unclear. The diagnosis is based on immunohistopathology, which is positive for human chorionic gonadotropin (hCG). It is a highly invasive and rapidly-disseminated hematogenous neoplasm, which leads to a poor prognosis. We present the case of a 57-year-old woman with upper gastrointestinal bleeding associated with weight loss. The gastroscopy showed, on the cardia and paracardial region, a $5 \times 4-\mathrm{cm}$ ulcerated polypoid lesion with circumscribed edges and without adjacent wall infiltration, with stigmas of recent bleeding and a pathological anatomy that demonstrates ulcerated pleomorphic malignancy with positive immunohistochemistry for pankeratin. Given the suspicion of poorly differentiated carcinoma and without evidence of metastasis, the patient underwent a total gastrectomy and the pathology was compatible with choriocarcinoma. Therefore, after the surgery, a serum hCG test was performed with a result of $714 \mathrm{mIU} / \mathrm{ml}$, which further confirmed the diagnosis. Treatment with adjuvant chemotherapy was decided, and a tomographic and serological hCG follow-up was conducted, without the presence of active disease.

Keywords: Stomach neoplasms; Choriocarcinoma; Gastrectomy; Chorionic gonadotropin; Surgery (Source: MeSH NLM).

1. Instituto de Enfermedades Neoplásicas. Lima, Perú.

2. Hospital Santa Rosa. Lima, Perú.

3. Hospital María Auxiliadora. Lima, Perú.

a. Médico Asistente de Servicio de Gastroenterología.

b. Médico Residente del Servicio de Gastroenterología.

c. Médico Asistente del Departamento de Patología.

d. Médico Asistente del Departamento de Abdomen.

* Autor corresponsal. 


\section{INTRODUCCIÓN}

Los coriocarcinomas son tumores de células trofoblásticas que habitualmente aparecen en las gónadas y suelen presentarse durante el embarazo (1). Sin embargo, se han descrito casos en los que se han visto comprometidos órganos extragonadales como pulmón, hígado, mama, vejiga, próstata y tracto gastrointestinal. Dentro de estos, el coriocarcinoma gástrico primario (CGP) alcanza una incidencia del $0,08 \%(1,2)$. Este tumor presenta una sintomatología similar al adenocarcinoma gástrico pero, al ser más vascularizado, puede manifestarse principalmente con hemorragia gastrointestinal (2). El diagnóstico se basa en el análisis inmunohistopatológico que, sin embargo, es positivo en la minoría de los casos ${ }^{(3)}$. Presentamos el caso de una paciente que cursa con hemorragia digestiva alta y que presenta, en la endoscopía, evidencia de lesión con características macroscópicas de un cáncer gástrico avanzado proliferativo con aspecto polipoideo y ulcerado (like Borrmann I ulcerado). Además, se definen características microscópicas compatibles con carcinoma poco diferenciado. La paciente es sometida a gastrectomía total y luego de la evaluación histológica se realiza el diagnóstico final de coriocarcinoma gástrico.

\section{CASO CLÍNICO}

Paciente mujer de 57 años, natural y procedente de Cusco (Perú), sin antecedentes de importancia. Acude a hospital de dicha región, con un tiempo de enfermedad de 7 semanas caracterizado por hematemesis, melena, epigastralgia, astenia y pérdida de peso. En el examen físico se observa piel pálida, funciones vitales normales y el resto de la evaluación sin hallazgos significativos. En los exámenes de laboratorio se encuentra hemoglobina $7 \mathrm{mg} / \mathrm{dl}$, glóbulos rojos microcíticos e hipocrómicos, y perfiles bioquímico y hepático dentro de los valores normales. Se realiza endoscopía digestiva alta en la que se evidencia lesión gástrica ulcerada sangrante con anatomía patológica compatible con adenocarcinoma. Se decide su referencia a Lima a hospital de mayor complejidad.

Al momento de la referencia al Instituto Nacional de Enfermedades Neoplásicas (INEN), la paciente presenta nuevo cuadro de hematemesis, por lo que se decide repetir la gastroscopia, y se encuentra una lesión polipoidea ulcerada de $5 \times 4 \mathrm{~cm}$ de superficie necrótica, con sangrado espontáneo focal a nivel de cardias y región paracardial. Se realiza biopsia que informa neoplasia maligna pleomórfica ulcerada (Figura 1) con inmunohistoquímica positiva para panqueratina, lo que hizo sospechar de carcinoma poco diferenciado. Se complementa estudio con tomografía axial computarizada de cerebro, tórax, abdomen y pelvis, sin presencia de metástasis.

Con dicho diagnóstico, se realiza gastrectomía total radical D2 (Figura 2), con informe microscópico de neoplasia maligna pleomórfica sin evidencia de componente de adenocarcinoma asociado. Ante la sospecha de coriocarcinoma, se solicita inmunohistoquímica, con resultado negativo para alfa feto proteína (AFP) y positiva para panqueratina y hCG (Figura $3 \mathrm{~A}-\mathrm{B}-\mathrm{C}$ ), lo que confirmó el diagnóstico de CGP.

Después de la cirugía y con el diagnóstico histológico en referencia, se realiza estudio de hCG sérica $(714 \mathrm{mIU} /$ $\mathrm{ml})$. Paciente recibió quimioterapia y fue evaluada periódicamente. Actualmente, permanece asintomática con controles de hCG inferiores a $2 \mathrm{mIU} / \mathrm{ml}$ y tomografía axial computarizada de cerebro, tórax, abdomen y pelvis sin evidencia de enfermedad ni metástasis a distancia luego de 2 años de la cirugía.

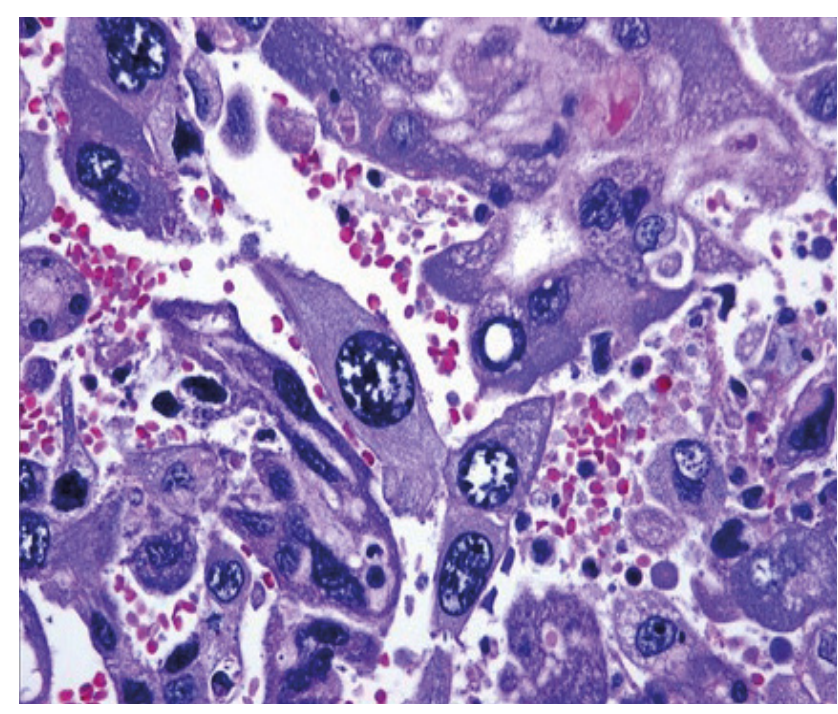

Figura 1. Informe anatomopatológico de biopsia endoscópica: células pleomórficas con inclusiones citoplasmáticas

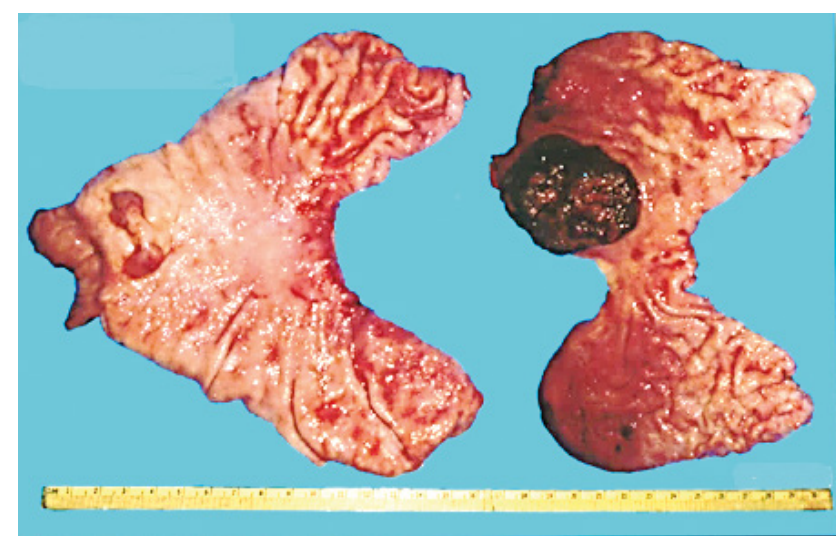

Figura 2. Coriocarcinoma de 5,0 cm localizado a nivel de cardias y región para-cardial, asociado a extensa hemorragia y necrosis central 


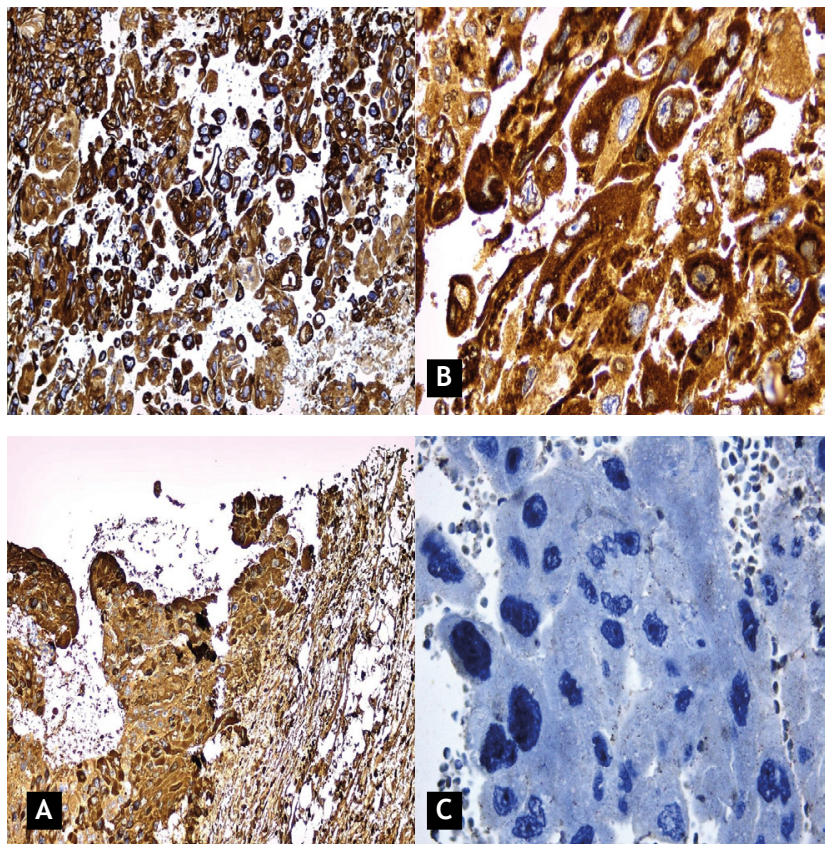

Figura 3. Inmunohistoquímica. (a) Panqueratina positiva (AE1/ AE3). (b) hCG positiva. (c) AFP negativa

\section{DISCUSIÓN}

En 1905, Davidsohn describe el primer caso de CGP $(1,2)$. Este es un tumor muy raro $(<1 \%$ neoplasias gástricas malignas) y de mal pronóstico ${ }^{(4)}$, por lo que, hasta la fecha, se han reportado a nivel mundial alrededor de 140 casos ${ }^{(2)}$. Además, la mayoría se caracteriza por ser tumores compuestos, asociados con adenocarcinoma pobremente diferenciado $(70 \%)$ y, muy raramente, son coriocarcinomas puros $(25 \%)^{(5)}$.

En el Perú, hasta la fecha, se ha realizado un estudio en el INEN, que es un centro de referencia para pacientes oncológicos. Este trabajo abarcó un periodo de 50 años, en ese tiempo se admitieron 7445 pacientes con cáncer gástrico (con estudio patológico del reporte quirúrgico confirmado), de ellos, solo 2 presentaron coriocarcinoma (6) (Figura 4).

Esta enfermedad es más frecuente en varones (relación de 2,3:1 respecto a las mujeres), y la edad media de presentación es de 60 años ${ }^{(5,7)}$. Los tumores suelen ser exofíticos, rojizos y se localizan en el tercio inferior del estómago ${ }^{(5,8)}$. Por otro lado, aunque no existen signos ni síntomas patognomónicos, la presentación más frecuente es la hemorragia gastrointestinal. Además, al momento del diagnóstico, la mayoría presenta metástasis ${ }^{(5,8)}$. Sin embargo, en nuestro caso, esta no se evidenció.

\section{CÁNCER GÁSTRICO HISTORIA NATURAL DIAGNÓSTICO Y RESULTADOS DE TRATAMIENTOS \\ INEN 1950 - 1999}

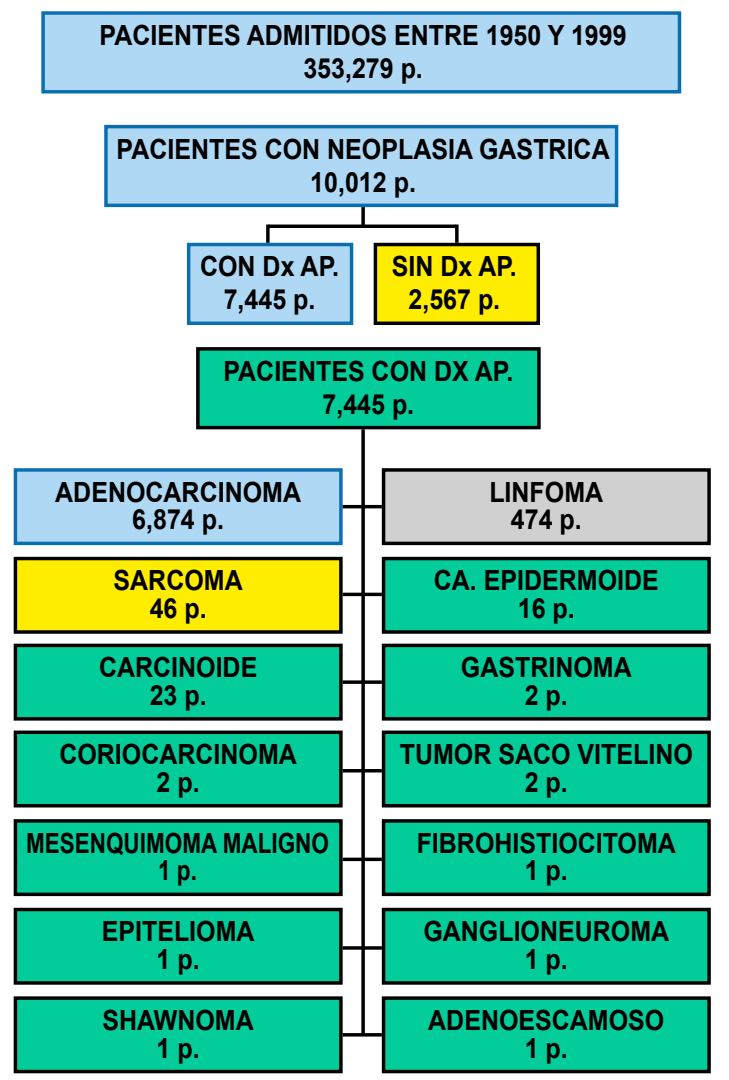

Figura 4. Cáncer gástrico: Historia natural, diagnóstico y resultados de tratamiento ${ }^{(6)}$

Existen algunas teorías sobre el mecanismo por el cual se desarrolla el coriocarcinoma gástrico. Una de ellas se basa en la metástasis de una lesión primaria genital que es diagnosticada tardíamente ${ }^{(4)}$. Por otro lado, Hartz y Ramírez, creían que este tumor se originaba a partir de un teratoma gástrico ${ }^{(9)}$. Otra teoría, iniciada por Pick en 1926 , se basa en la desdiferenciación de las células malignas del adenocarcinoma gástrico al nivel del ectodermo embrionario, que conservan la capacidad de formar trofoblastos. Esta hipótesis se plantea debido a que la mayoría de coriocarcinomas se asocian a adenocarcinoma, y que, en algunos casos, se ha demostrado que existe una transición entre ambos. Además, en los coriocarcinomas gástricos se encuentra una combinación de citotrofoblastos malignos y sincitiotrofoblastos con áreas de diferenciación glandular típica, lo que respalda la hipótesis de la retrodiferenciación. Por lo tanto, el coriocarcinoma puro surgiría a través del crecimiento excesivo y la eliminación del adenocarcinoma original. Esta última teoría es la más aceptada, y ha sido respaldada por Krulewski en $1988^{(3,9,10)}$. 
La presencia de marcadores tumorales específicos facilita el diagnóstico. La elevación sérica de hCG se encuentra en el $100 \%$ de los coriocarcinomas y es altamente característica de esta estirpe histológica. Cabe resaltar que la hCG sérica es útil para evaluar la respuesta al tratamiento y la recidiva tumoral $(11,12)$.

En cuanto a la inmunohistoquímica, todos los tipos de células trofoblásticas, así como las áreas de diferenciación glandular, son positivas para la citoqueratina. Además, las células tumorales trofoblásticas típicamente muestran una inmunoreactividad fuerte para hCG ${ }^{(12,13)}$. En el caso de la AFP, lo que se pretende identificar es la presencia conjunta de adenocarcinoma ${ }^{(14)}$. Por ello, es que el caso presentado es un coriocarcinoma puro, pues la inmunoreactividad para AFP fue negativa.

Pese a que el tratamiento de elección no está claramente definido, se sugiere manejo multidisciplinario, que consiste en gastrectomía total con disección de ganglios linfáticos, asociada a quimioterapia adyuvante. Sin embargo, el régimen de quimioterapia aún no ha sido estandarizado ${ }^{(15,16)}$. En comparación con el adenocarcinoma gástrico, el pronóstico del coriocarcinoma es más agresivo, debido a su comportamiento y rápida diseminación. El promedio de sobrevida suele ser menor de 2 meses ${ }^{(15)}$. Pese a ello, en el caso reportado, la paciente cursa con una sobrevida mayor a dos años.

Para concluir, debemos reiterar que, hasta el momento, la mayor información sobre esta patología se basa en reporte de casos. Como se mencionó, en el Perú existe un estudio general sobre cáncer gástrico en el que se reportan dos casos de CGP en 50 años, por lo que es importante documentar el presente caso. Además, pese a que esta enfermedad tiene generalmente un mal pronóstico, la paciente tuvo una sobrevida mayor a la esperada. Asimismo, es probable que existan variables predictoras de buen pronóstico que aún no han sido estudiadas y esto motivará a realizar estudios epidemiológicos, no obstante, lo poco frecuente del cuadro, para realizar tamizaje en grupos de riesgo, diagnóstico precoz y tratamiento para así lograr una opción curativa.

\section{REFERENCIAS BIBLIOGRÁFICAS}

1. Baraka BA, Al Kharusi SS, Al Bahrani BJ, Bhathagar G. Primary gastric chorioadenocarcinoma. Oman Med J. 2016;31(5):381-3.

2. Kobayashi A, Hasebe T, Endo Y, Sasaki S, Konishi M, Sugito M, et al. Primary gastric choriocarcinoma: two case reports and a pooled analysis of 53 cases. Gastric Cancer. 2005;8(3):178-85.

3. Krulewski T, Cohen LB. Choriocarcinoma of the stomach: pathogenesis and clinical characteristics. Am J Gastroenterol. 1988 Oct;83(10):1172-5.

4. Shastri A, Daver NG, Hayes TG. Primary gastric chorioadenocarcinoma: a needle in a haystack. Rare Tumors. 2011 April 4;3(2):56-8.

5. Yoon JH, Kim MS, Kook EH, Ahn SH, Jeong SY, Han MS, et al. Primary gastric choriocarcinoma: two case reports and review of the literatures. Cancer Res Treat. 2008 Sep;40(3):145-50.

6. Ruiz E, Barreda F, Celis J, et al. Cáncer Gástrico: Historia Natural Diagnóstico y Resultados de Tratamiento. INEN 1950 -1999. Informe Final del Proyecto de Investigación Financiado por CONCYTEC. 2002.

7. Liu Z, Mira JL, Cruz-Caudillo JC. Primary gastric choriocarcinoma: a case report and review of the literature. Arch Pathol Lab Med. 2001 Dec;125(12):1601-4.

8. Raghavapuram R, Veerankutty FH, Anandakumar M. Primary Choriocarcinoma of the Stomach. A Case Report and Review of the Literature. Indian J Surg Oncol. 2016 Mar;7(1):119-23.

9. Unverdi H, Savas B, Ensari A, Akgül H. Unusual tumor: Primary gastric choriocarcinoma. Turk J Gastroenterol. 2011 Aug;22(4):437-39.

10. Pai MR, Mathai AM, Kumar S, Prabhu S. Coexistent gastric primary choriocarcinoma and adenocarcinoma. Indian J Pathol Microbiol. 2009;52(4):537-39.

11. Noguchi T, Takeno S, Sato T, Takahashi Y, Uchida Y, Yokoyama S. A patient with primary gastric choriocarcinoma who received a correct preoperative diagnosis and achieved prolonged survival. Gastric Cancer. 2002;5(2):112-17.

12. Yakeishi Y, Mori M, Enjoji M. Distribution of beta-human chorionic gonadotropin-positive cells in noncancerous gastric mucosa and in malignant gastric tumors. Cancer. 1990 Aug 15;66(4):695-701.

13. Saigo PE, Brigati DJ, Sternberg SS, Rosen PP, Turnbull AD. Primary gastric choriocarcinoma. An immunohistological study. Am J Surg Pathol. 1981 Jun;5(4):333-42.

14. Eom BW, Jung S-Y, Yoon H, Kook M-C. Gastric choriocarcinoma admixed with an a-fetoprotein-producing adenocarcinoma and separated adenocarcinoma. World J Gastroenterol. 2009 Oct 28;15(40):5106-8.

15. Takahashi K, Tsukamoto S, Saito K, Ohkohchi N, Hirayama K. Complete response to multidisciplinary therapy in a patient with primary gastric choriocarcinoma. World J Gastroenterol. 2013 Aug 21;19(31):5187-94.

16. Schmoll HJ, Souchon R, Krege S, Albers P, Beyer J, Kollmannsberger $C$, et al. European consensus on diagnosis and treatment of germ cell cancer: a report of the European Germ Cell Cancer Consensus Group (EGCCCG). Ann Oncol. 2004 Sep;15(9):1377-99.

Fuentes de financiamiento

Este artículo ha sido financiado por los autores.

Conflictos de interés

Los autores declaran no tener ningún conflicto de interés.

Correspondencia:

Fernando Barreda Bolaños

Dirección: Scipion Llona $\mathrm{N}^{\circ} 180$ Of 603, Miraflores. Lima, Perú.

Teléfono: 998094949

Correo electrónico: Fernando.barreda73@hotmail.com

Recibido: 05 de noviembre de 2018.

Evaluado: 30 de enero de 2019. Aprobado: 01 de febrero de 2019.

(c) La revista. Publicado por Universidad de San Martín de Porres, Perú. (cc) Br Licencia de Creative Commons Artículo en acceso abierto bajo términos de Licencia Creative Commons Atribución 4.0 Internacional. (http://creativecommons.org/licenses/by/4.0/)

\section{ORCID iDs}

Fernando barreda Bolaños Humberto Liu Bejarano Jéssica Alférez Andía Roxana Inoñan García Henry Guerra Miller Eduardo Payet Meza https://orcid.org/0000-0002-7923-6299 https://orcid.org/0000-0002-8417-0837 https://orcid.org/0000-0002-1314-1422 (i) https://orcid.org/0000-0002-8866-915X (iD) https://orcid.org/0000-0002-4894-5631

(iD) https://orcid.org/0000-0001-9434-3888 